\title{
Modulation of nuclear receptor Liver X Receptor alpha by polyphenols
}

\author{
K. Nugraheni, S.A. Hutchinson, J.L. Thorne and C. Boesch \\ School of Food Science and Nutrition, Nutrition and Public Health Group, University of Leeds, LS2 9JT.
}

Polyphenols comprise a large group of plant secondary metabolites that have been associated with numerous beneficial effects to promote and maintain health and thereby prevent the onset of a number of diseases such as diabetes, cardiovascular disease and cancer. ${ }^{(1,2)}$. The detailed mechanisms by which polyphenols act on a cellular level are still largely unknown. It has recently been suggested that some members of the flavonoid subgroup of polyphenols, might have the ability to act as ligands for nuclear receptors and thereby impact on transcriptional regulation of metabolic pathways, such as cell development, energy metabolism and inflammation $^{(3)}$. The aim of this study was to investigate the effects of different polyphenols on activation of the nuclear receptor LXR $\alpha$. LXR $\alpha$ is the primary regulator of cholesterol and fatty acid metabolism. Activation of LXR $\alpha$ also inhibits pro-inflammatory gene expression $^{(4)}$.

Human breast cancer cells, stably transfected with LXR $\alpha$ /luciferase reporter, were incubated with the polyphenols hesperetin (flavanone), quercetin and isorhamnetin (flavonol), resveratrol (stilbene), genistein (isoflavone), epigallocatechin gallate (flavanol) in increasing concentrations $(5-50 \mu \mathrm{M})$ and T0901417 $(1 \mu \mathrm{M})$ as positive control for 16 hours. LXR $\alpha$ activation was assessed using luciferase assay and normalised to total cell protein measured using BCA assay. The neutral red assay was conducted to evaluate cytotoxicity of polyphenols. Data were analyzed using one way ANOVA followed by Dunnett's posthoc test.

Hesperetin, quercetin, isorhamnetin, resveratrol, and genistein demonstrate capacity to activate LXR $\alpha$ albeit to a differing degree with genistein providing most consistent activation as indicated in Fig 1. EGCG showed very weak LXR $\alpha$ activation. None of the test compounds and solvent caused cytotoxicity (viability $>80 \%$ ) in the concentrations applied (data not shown).
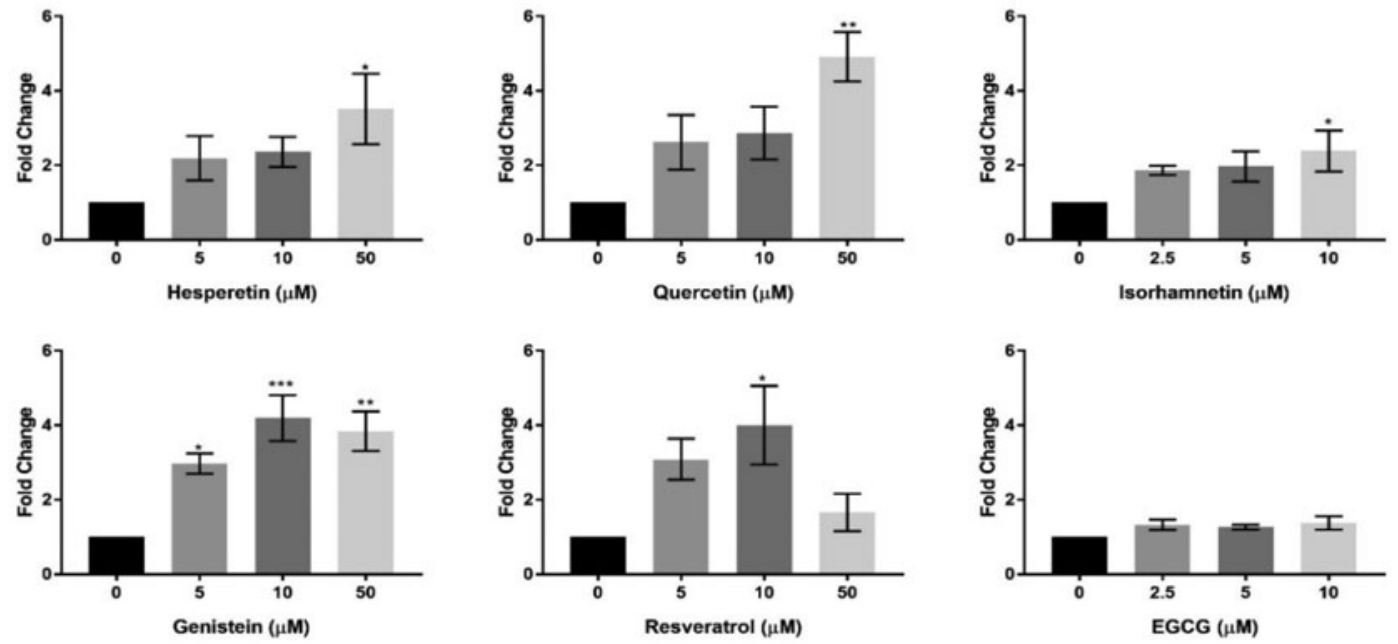

Fig. 1. Effects of increasing concentrations of different polyphenols on LXR $\alpha$ activation. Cells were incubated for 16 hours and luciferase activity was normalized to total cell protein. Data are presented as mean with SEM from three independent experiments performed in triplicate. * indicate significant differences to solvent control.

Our results indicate that polyphenols belonging to different subclasses can activate LXR $\alpha$ and further studies are needed to demonstrate effects on LXR $\alpha$ target gene expression and metabolic changes including application in more relevant cell types such as liver cells.

1. Zhang H, Tsao R (2016) Curr Opin Food Sci 8, 33-42.

2. Croft KD. (2016) Arch Biochem Biophys 595, 120-124.

3. Avior Y, Bomze D, Ramon O \& Nahmias Y (2013) Food Funct 6, 831-844.

4. Viennois E et al. (2012) Mol Cell Endocrinol 351, 129-141. 